\title{
TMEEP: Threshold-sensitive based Multihop Energy Efficient Protocol for HWSN
}

\author{
Muhammad Rizwan ${ }^{1,}$, Muhammad S. Nisar ${ }^{2, b}$ and Hongbo Jiang ${ }^{1, c}$ \\ ${ }^{1}$ School of Electronic Information and Communications, HUST, Wuhan 430074, China; \\ ${ }^{2}$ Wuhan National Laboratory for Optoelectronics, HUST, Wuhan 430074, China. \\ aI201422058@hust.edu.cn, 'b1201322185@hust.edu.cn, chongbojiang@hust.edu.cn
}

Keywords: Wireless Sensor Network (WSN), Base Station (BS), Throughput, Network Lifetime.

\begin{abstract}
WSNs are expected to find wide applicability in various fields ranging from engineering industry to our home environment technology. Efficient utilization of energy of sensor nodes and short distance multihop communication are the major factors in order to reduce the energy consumption in a sensor network. In this research work, we have proposed a new protocol; Threshold-sensitive based Multihop Energy Efficient Protocol (TMEEP) for Heterogeneous Wireless Sensor Network. TMEEP is a reactive protocol using three level of heterogeneity. TMEEP combines the idea of threshold sensitive based data collection and short distance multihop communication for achieving the best performance in terms of energy consumption and network lifetime. We evaluate our routing protocol for a simple sensing application and compare results with some other existing protocols like SEP-E and LEACH. The simulation results indicate that proposed scheme enhanced the stability period, network lifetime and throughput than existing routing protocols.
\end{abstract}

\section{Introduction}

Recent advances in Micro-Electro-Mechanical Systems (MEMS) technology help in the development of smart sensors with sensing, processing and communication capabilities. WSNs have a large number of smart sensor nodes that are deployed densely to monitor a physical or environment conditions. The sensor nodes have the ability to sense, gather and measure the information and transmitted the collected data to sink. There are many application of WSN which includes industrial surveillance, home automation and environmental monitoring [1]. Heterogeneous WSNs have become more popular because it takes full advantage of heterogeneity in order to meet the demands of various applications which have been studied in recent literature [2].

The major crucial challenges in the organization of WSN are energy efficiency, stability period of network and enhancement in network life time. Since sensor nodes are battery operated devices and changing or recharging of battery is very difficult due to their dense deployment in the remote harsh environment. Thus, it is important to introduce the novel energy efficient routing protocols that ensure the efficient utilization of energy of sensors in order to extend the life time of WSNs. [3].

Clustering is an important mechanism in order to enhance the stability of network. Clustering has been widely used in various sensor applications. Cluster based routing schemes are very helpful by reducing the cost of data aggregation and transmission of sensed data before transmitting to sink [4].

\section{Heterogeneous and Energy Dissipation Model}

This model comprises of n number of sensor nodes randomly deployed in MxM area. BS is located in the middle of sensing field. There are three types of nodes with different energy i.e. normal $n$, advance $\mathrm{m}$, and super nodes mo. Super nodes have more initial energy than advance nodes and advance nodes have more energy than normal nodes. Let $E_{0}$ is the initial energy of each normal node , $(1+\alpha) \cdot E_{0}$ is for advance node and $(1+\beta) \cdot E_{0}$ is for super nodes. Total energy of the system is increased by a factor $(1+\mathrm{m}(\alpha+\operatorname{mo} . \beta))$. Total initial energy $E_{\text {total }}$ of HWSN model is given by: $n \cdot E_{0}(1-m)+n \cdot m(1-m o)(1+\alpha) E_{0}+n \cdot m \cdot m o \cdot E_{0}(1+\beta)=n \cdot E_{0}(1+m(\alpha+m o \cdot \beta))(1)$ 
In this work, we use the radio energy dissipation model similar to [5]. Free space and multipath fading channel are used in this model. The amount of energy required to transmit $\mathrm{T}$ bits message over a distance $\mathrm{d}$ from one node to another node is given by:

$$
E_{T x}(T, d)=\left\{\begin{array}{l}
T \times \text { Electr }+T \times E_{f s} \times d^{2} \text { if } d<d_{0} \\
T \times \text { Electr }+T \times E_{m p} \times d^{4} \text { if } d<d_{0}
\end{array}\right.
$$

Electr is the dissipated energy to run the transmitter or receiver. The parameters $E_{f s}$ and $E_{m p}$ is the amount of energy dissipation which depends upon the distance $\mathrm{d}_{0}$ which is given as $d_{0}=\sqrt{E_{f s} / E_{m p}}$. To receive the $\mathrm{T}$ bits message, dissipated energy is equal to $E_{r x}(T)=T$. $E_{r x-e l e c}$.

\section{TMEEP: Threshold-sensitive based Multihop Energy Efficient Protocol}

In TMEEP, $\mathrm{n}$ sensor nodes are randomly dispersend in the $\mathrm{M} \times \mathrm{M}$ sensing region. The distance from node to $\mathrm{CH}$ or sink is $\leq \mathrm{d}_{0}$. The energy dissipated by $\mathrm{CH}$ and non-CH in a single round is defined as:

$$
\begin{aligned}
& E_{C H}=T . E \text { Eectr }\left(\frac{n}{k}-1\right)+T \cdot E_{D A} \frac{n}{k}+T \cdot \text { Electr }+T \cdot E_{f S} d_{t o B S}^{2} \\
& E_{\text {nonCH }}=T .\left(\text { Electr }+E_{f s} \times d_{t o C H}^{2}\right)
\end{aligned}
$$

Where $E_{D A}$ is defined as the energy required for data aggregation in a round, $\mathrm{k}$ is defined as number of clusters, $d_{t o C H}$ is defined as the average distance between nodes and $\mathrm{CH}$ and $d_{t o B S}$ is defined as average distance between cluster head and base station. Where. The total expended energy can be calculated as follows:

$$
E_{\text {total }}=T .\left(2 n E_{\text {elec }}+n E_{D A}+k E_{f S} d_{\text {toBS }}^{2} \times n E_{f s} d_{\text {toCH }}^{2}\right)
$$

The optimal number of clusters can be calculated by $k_{\text {opt }}=\sqrt{E_{f s} / E_{m p}} \times \sqrt{n / 2 \pi} \times \sqrt{M / d_{\text {toBS }}^{2}}$ the average distance $d_{t o B S}$ can be calculated as follows [6] $d_{t o B S}=0.765 \times M / 2$ and the optimal probability of node to become a $\mathrm{CH}$ in a round is defined as $p_{\text {opt }}=k_{\text {opt }} / n$. Let $p_{n r m l}, p_{a d v}$ and $p_{s p r}$ represents the weighted election probability for normal, advance and super nodes. The weighted probability of normal and advance nodes can be calculated as:

$$
p_{\text {nrml }}=\frac{p_{\text {opt }}}{(1+m(\alpha+m o . \beta))}, p_{a d v}=\frac{p_{o p t} \times(1+\alpha)}{(1+m(\alpha+m o . \beta))}, p_{\text {spr }}=\frac{p_{o p t} \times(1+\beta)}{(1+m(\alpha+m o . \beta))}
$$

In the two-level HWSN, we replace the reference $p_{\text {opt }}$ value with the weighted probabilities given in Eq. 6. Therefore, $p_{i}$ is changed into:

$$
p_{i}=\left\{\begin{array}{l}
\frac{p_{o p t} E_{i}(r)}{(1+m(\alpha+m o . \beta)) \bar{E}(r)} \text { if } S_{i} \text { is normal node } \\
\frac{p_{o p t} E_{i}(r)(1+\alpha)}{(1+m(\alpha+m o . \beta)) \bar{E}(r)} \text { if } S_{i} \text { is advance node } \\
\frac{p_{o p t} E_{i}(r)(1+\beta)}{(1+m(\alpha+m o . \beta)) \bar{E}(r)} \text { if } S_{i} \text { is super node }
\end{array}\right.
$$

The T $\left(S_{i}\right)$ probability threshold by which node $S_{i}$ uses to determine whether it can be elected as $\mathrm{CH}$ in a round is defined as:

$$
\mathrm{T}\left(S_{i}\right)=\left\{\begin{array}{cl}
\frac{p_{i}}{1-p_{i}\left(r \bmod 1 / p_{i}\right)} & \text { if } S_{i} \in Q^{\prime} \\
0 & \text { otherwise }
\end{array}\right.
$$

Where $Q^{\prime}$ is defined as the set of either normal, advance or super nodes that are not elected as CHs within the last $1 / p_{n r m l}, 1 / p_{a d v}$ or $1 / p_{s p r}$ rounds.

Proposed TMEEP: In this research work, we have proposed a new protocol; Threshold-sensitive based Multihop Energy Efficient Protocol (TMEEP) for Heterogeneous Wireless Sensor Network. TMEEP is a reactive protocol using three level of heterogeneity. TMEEP is a reactive protocol using three level of heterogeneity. TMEEP combines the idea of threshold sensitive based data collection and short distance multihop communication for achieving the best performance in terms of energy consumption and network lifetime. In TMEEP, sensor nodes keep on sensing and transmit data only if a specific threshold is reached. TMEEP uses residual energy based election of CHs with double layered election probability mechanism. 


\section{Simulation and Results Analysis}

In this section, we evaluate the performance of purposed routing scheme. The following performance parameters are used to evaluate the performance of purposed routing scheme.

Stability period. Stability Period is the time interval from when the network operation starts until death of first node.

Instability period. Instability Period is the time interval starting from the death of $1^{\text {st }}$ node till death of last node.

Network lifetime. Network lifetime is the time interval between the network operation start until the dear of last node.

Throughput. This will measure the total number of packets which are sent to base station.

We compare it with LEACH and SEP-E [7] in the same heterogeneous setting. The packet size is 500 bytes and initial energy is $E_{0}=0.5 \mathrm{~J}$. The other key parameters used in simulation are given in Table 1.

Table 1 Simulation Parameters

\begin{tabular}{cl}
\hline Parameter & Value \\
\hline Area & $100 \mathrm{~m} \times 100 \mathrm{~m}$ \\
$N$ & 100 \\
$E_{\text {elec }}$ & $50 \mathrm{~nJ} / \mathrm{bit}$ \\
$E_{D A}$ & $5 \mathrm{~nJ} / \mathrm{bit} / \mathrm{message}$ \\
$E_{m p}$ & $10 \mathrm{pJ} / \mathrm{bit} / \mathrm{m}^{4}$ \\
$E_{f s}$ & $0.0013 \mathrm{pJ} / \mathrm{bit} / \mathrm{m}^{2}$ \\
$P_{\text {opt }}$ & 0.1 \\
$d_{o}$ & $87.7 \mathrm{~m}$ \\
\hline
\end{tabular}

We performed the simulations for $\mathrm{m}=0.1, \mathrm{~m} 0=0.1, \mathrm{a}=1, \mathrm{~b}=2$ and rounds $=15000$. According to Table 2, we can see that TMEEP has enhanced the stability period and network lifetime of the network as compared to LEACH and SEP-E. We can observe that in LEACH and SEP-E, the death of first node occurred at $504^{\text {th }}$ round and $1092^{\text {nd }}$ round while in TMEEP the death of first node occurred at $2001^{\text {st }}$ round. We can verify that the stability period and network life time of network is improved in the case of TMEEP as compare to LEACH and SEP-E because in TMEEP, sensor nodes keep on sensing and transmits data only if a specific threshold is reached.

Table 2 Comparison Table

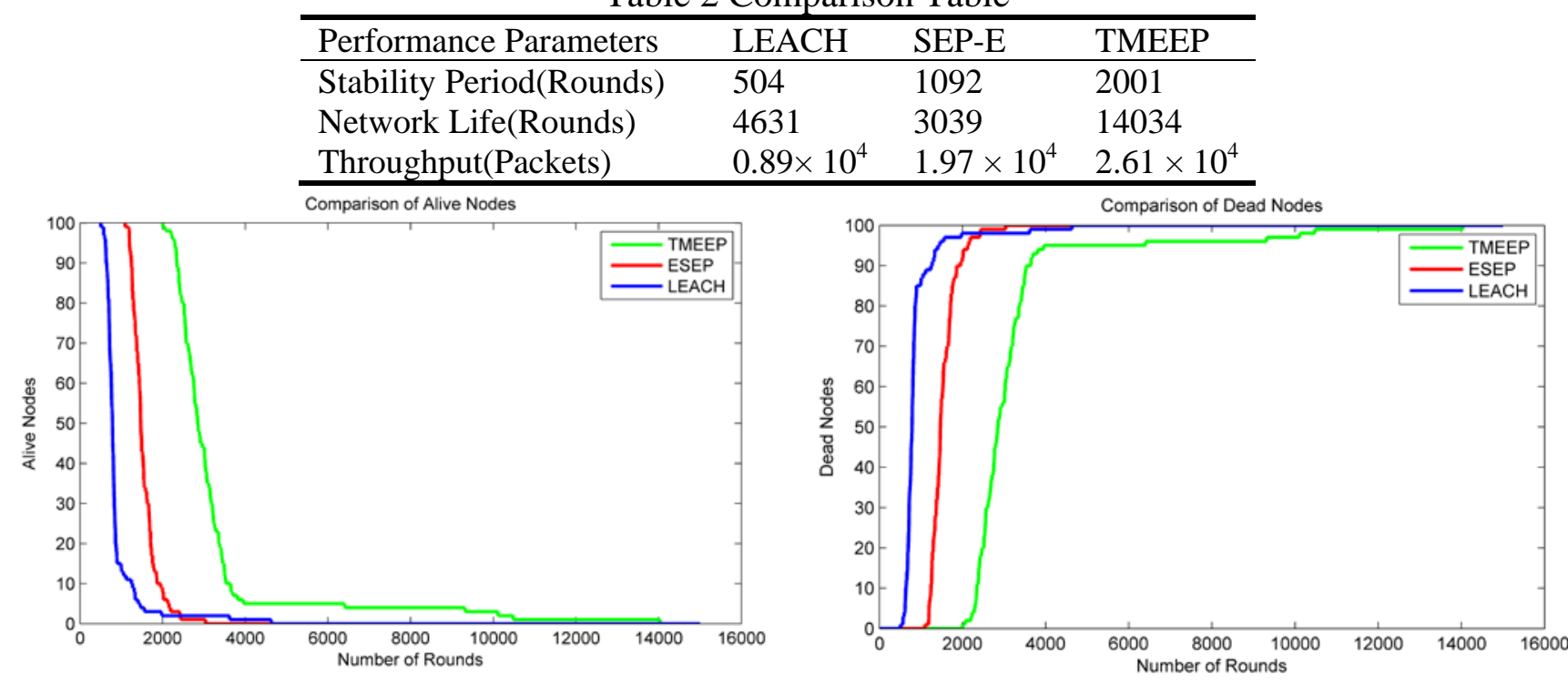

Fig. 1. Comparison of Alive Nodes

Fig. 2. Comparison of Dead Nodes

In Fig. 1, we can observe that TMEEP has increased the stability period of the network as compared to LEACH and SEP-E. We can validate that TMEEP has significantly increased the instability period of the network as compared to LEACH and SEP-E as shown in TABLE II. In LEACH and SEP-E all nodes died at $5064^{\text {th }}$ round and $3081^{\text {th }}$ round respectively while in TMEEP all nodes died at 10918 rounds. It is thus clear that the network lifetime has improved in the case of 
TMEEP as compare to LEACH and SEP-E as shown in Fig. 2. In Table 2, we can see that TMEEP has enhanced the throughput of the network as compared to LEACH and SEP-E. Fig. 3 shows the throughput of TMEEP is higher than LEACH and SEP-E. The results show that the total number of packets sent from CHs to sink is higher in the case of TMEEP as compared to LEACH and SEP-E. Fig. 4 shows the throughput of TMEEP is higher than LEACH and SEP-E for following parameters, $\mathrm{m}=0.1, \mathrm{~m} 0=0.2, \mathrm{a}=0.5, \mathrm{~b}=1$ and rounds $=12000$. Our simulation results prove that the throughput of TMEEP is higher than LEACH and SEP-E. Thus, it is clearly concluded that throughput is significantly enhanced in the case of TMEEP as compared to LEACH and SEP-E.

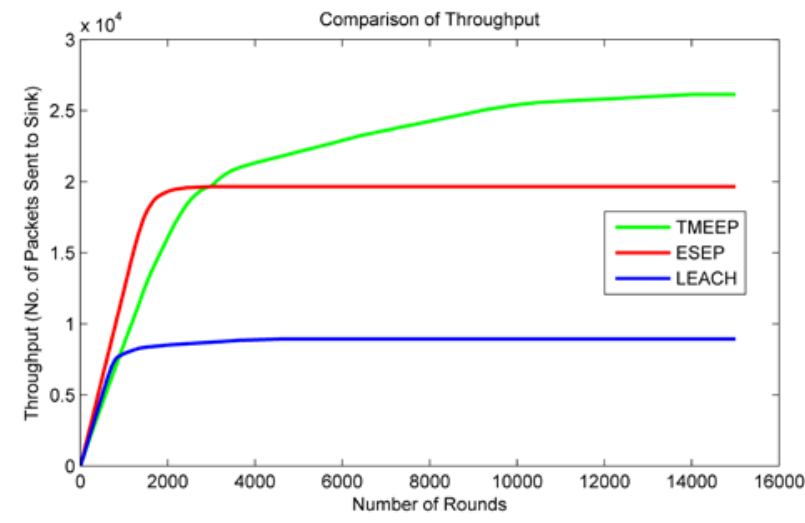

Fig. 3. Comparison of Throughput

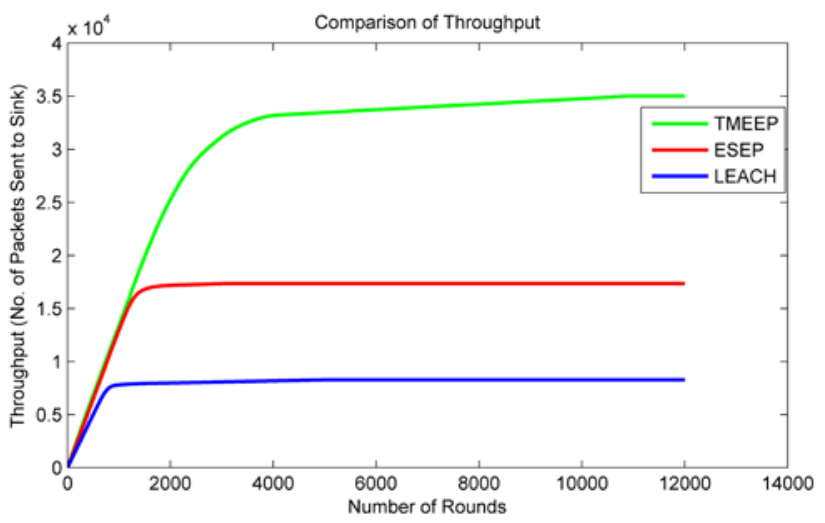

Fig. 4. Comparison of Throughput

\section{Conclusion}

In this research work, we proposed TMEEP a reactive routing protocol for heterogonous wireless sensor network. TMEEP is a threshold based energy efficient routing protocol with an additional feature of three-level heterogeneity. TMEEP protocol performs best for time critical applications. The simulation results clearly proved that the purposed routing protocol, TMEEP, significantly enhanced the stability period, network life time and throughput of the sensor network. The simulation results indicate that the stability period of network is enhanced $83.2 \%$ approximately by reactive approach, means that data transmission is done only when a threshold is reached.

\section{References}

[1] I.F. Akyildiz, W.Su, Y. Sankarasubmmaiam, E.Cayirci, “A Survey on Sensor Networks,” IEEE Comm. Magazine, pp.102-114, Aug 2002.

[2] R Kumar, V. Tsiatsis, M.B. Srivastava, Computation Hierarchy for In-Network Processing, Proceedings of 2nd ACM International Workshop Applications, San Diego, CA, 68-77, 2003.

[3] Kemal Akkaya and Mohamed Younis, "A Survey on Routing Protocols for Wireless Sensor Networks,” Ad Hoc Networks, Vol. 3, No. 3, pp. 325-349, May 2005.

[4] J. Al-Karaki, and A. Kamal, "Routing Techniques in Wireless Sensor Networks: A Survey," IEEE Communications Magazine, Vol. 11, no. 6, Dec. 2004, pp. 6 -28.

[5] W. Heinzelman, A. Chandrakasan, and H. Balakrishnan, "Energy Efficient Communication Protocols for Wireless Microsensor Networks,” Proc. Of 33th Hawaiian International Conference on Systems Science, 2000.

[6] Smaragdakis, I Matta, A Bestavros. SEP: A Stable Election Protocol for clustered heterogeneous wireless sensor networks. in: Second International Workshop on Sensor and Actor Network Protocols and Applications (SANPA). 2004.

[7] Femi A. Aderohunmu, Jeremiah D. Deng, “ An Enhanced Stable Election Protocol (SEP) for Clustered Heterogeneous WSN.” Dept. Information Science, University of Otago, New Zealand. 